\title{
Complex Chiral Modulations in FeGe close to Magnetic Ordering
}

\author{
E. Moskvin,,${ }^{1,2}$ S. Grigoriev, ${ }^{1,2}$ V. Dyadkin, ${ }^{1}$ H. Eckerlebe, ${ }^{3}$ M. Baenitz,${ }^{4}$ M. Schmidt,${ }^{4}$ and H. Wilhelm ${ }^{5}$ \\ ${ }^{1}$ Petersburg Nuclear Physics Institute, NRC Kurchatov Institute, Gatchina, Saint-Petersburg, 188300, Russia \\ ${ }^{2}$ Saint-Petersburg State University, Ulyanovskaya 1, Saint-Petersburg, 198504, Russif* \\ ${ }^{3}$ Helmholz-Zentrum Geesthacht, 21502 Geesthacht, Germany \\ ${ }^{4}$ Max Planck Institute for Chemical Physics of Solids, 01187 Dresden, Germany \\ ${ }^{5}$ Diamond Light Source Ltd, Chilton, Didcot, Oxfordshire, OX11 ODE, United Kingdom
}

\begin{abstract}
We report on detailed polarized small-angle neutron scattering on cubic FeGe in magnetic fields applied either along (transverse) the scattering vector or parallel (longitudinal)to the neutron beam. The $(H, T)$ phase diagrams for all principal axes contain a segmented $A$-phase region just below the onset of magnetic order. Hexagonal Bragg-spot patterns were observed across the entire $A$-phase region for the longitudinal geometry. Scattering intensity was observed in parts of the A phase for both scattering configurations. Only in a distinct pocket $\left(A_{1}\right)$ vanishing scattering intensity was found in the transverse geometry.

PACS numbers: $75.25 .-\mathrm{j}, 75.30 . \mathrm{Kz}$
\end{abstract}

In noncentrosymmetric magnetic mono-silicides and mono-germanides the chiral Dzyaloshinskii-Moriya (DM) interaction 1 usually stabilizes spiral one-dimensional modulations [2]. Further theory developments have approved the stabilization of complex magnetic textures: $2 \mathrm{D}$ and $3 \mathrm{D}$ modulations with a fixed sense of rotation of the magnetization vector (chiral skyrmions) 3 6. Such skyrmions in a form of isolated and bound axisymmetric strings have been recently observed in thin films of metallic cubic helimagnets [7-10], in the cubic multiferroic $\mathrm{Cu}_{2} \mathrm{OSeO}_{3}$ 11 14, and in $\mathrm{Fe}$ mono atomic layers with surface/interface induced DM interactions [15]. In thin magnetic films chiral skyrmions represent areas of reverse magnetization localized into spots of nanoscale size [6, 7].

The observed spiral, conical and skyrmionic states in cubic helimagnets in a broad temperature $(T)$ and magnetic field $(H)$ range are described by rotational modes of the magnetization vectors with fixed magnitudes [1 4. In close vicinity of the Curie temperature $\left(T_{\mathrm{C}}\right)$ however, unconventional magnetic properties and numerous physical anomalies are observed [16 26 which are denoted as precursor effects 27, 28. They are explained theoretically by softening of the magnetization modulus and a strong coupling between angular and longitudinal modes near $T_{\mathrm{C}}$ [6, 27, 31]. Magnetic measurements revealed a small closed pocket, the so called $A$ phase, in the $(H, T)$ phase diagram of MnSi [16, 18, FeGe [27, 28, and some other cubic helimagnets 29, 30. Small-angle neutron scattering (SANS) experiments at selected $T$ showed that in this area magnetic modulations are transverse to the $H$ direction [20, 21] and can produce six-fold Bragg spot patterns [11, 22, 23].

This vast amount of experimental and theoretical studies provides considerable insight into the physical nature of the precursor states in cubic helimagnets. Particularly, it was conclusively established that the $A$-phase pocket includes a multiplicity of different modulated states with clear indications of phase transitions between them [27. This is now supported by recent findings in $\mathrm{Cu}_{2} \mathrm{OSeO}_{3}$ [11, 13. However, these results are still inconclusive to reconstruct detailed spin-structures arising in the $A$ phase area. This is mainly due to the fact that the SANS experiments were focussed on a single compound [21, 22] and more importantly, are restricted to a few particular $(H, T)$ points close to the low- $H$ and low- $T$ boundary of the $A$-phase, inferred from magnetic measurements [18]. There is no knowledge on the extension of the $A$-phase region based on SANS experiments. This important information, however, is needed to interpret SANS pattern from the edge of the $A$-phase region. Thus, only a careful investigation of the enigmatic $A$-phase region by neutrons can unveil the nature of the $H$-driven magnetic modulations.

In this Letter we report on detailed polarized SANS experiments on single crystals of FeGe as a function of external magnetic field at several temperatures below magnetic ordering. From this we were able to (i) determine the extension of the $A$-phase region, (ii) confirm the segmentation of the $A$ phase, (iii) establish a region were only longitudinal magnetic modulations exist, (iv) reveal a large phase space where also transverse modulations are present, and (v) confirm the $A$-phase boundaries deduced from magnetic measurements. These results unambiguously show that in FeGe and likely in some other cubic helimagnets the $A$-phase region is an assembly of several sub-pockets with rather complex 2D and 3D magnetic modulations.

A single crystal of the cubic polymorph of iron monogermanide, $\epsilon$-FeGe (B20-type of structure, space group $P 2_{1} 3$ ) [27] was studied using the SANS-2 instrument at the HZG, Geesthacht. The crystal was an irregular but almost spherical polyhedron with about $1.2 \mathrm{~mm}$ diameter. The polarized neutron beam had a wavelength $\lambda=$ $5.8 \AA(\Delta \lambda / \lambda=0.1)$ and an initial polarization $P_{0}=0.95$. The accessible $Q$-range was $0.003 \leq Q \leq 0.02 \AA^{-1}$. $H$ 


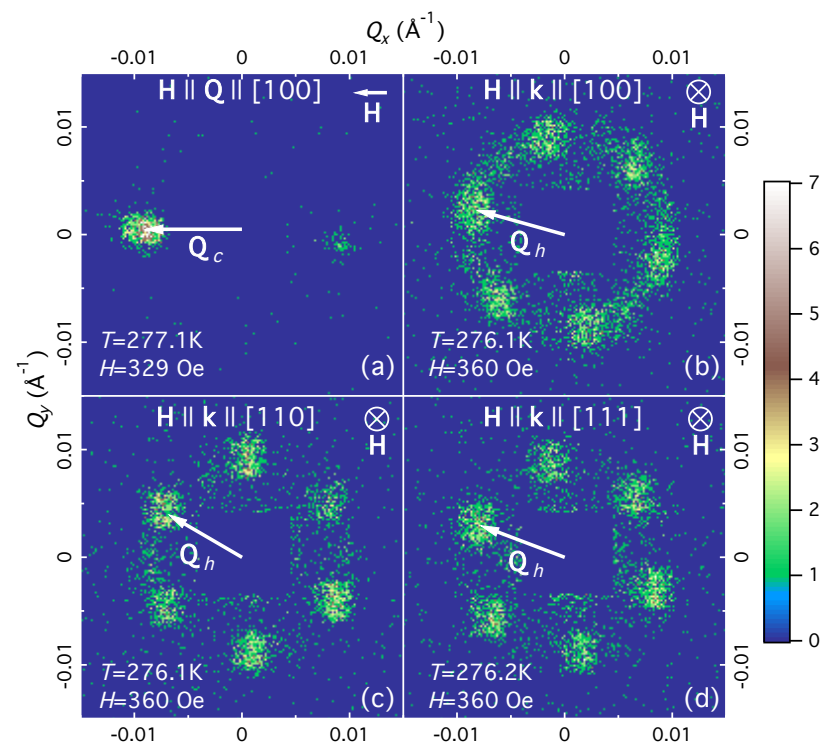

FIG. 1. (Color online) (a) SANS map for the transverse geometry with the polarization $\mathbf{P}_{0} \perp \mathbf{k}$, i.e. perpendicular to the incoming neutron beam, but parallel to $\mathbf{H}$ and the $\langle 100\rangle$ direction. (b-d) Representative scattering maps for the longitudinal geometry $\left(\mathbf{P}_{0} \| \mathbf{k}\right)$. Hexagonal Bragg-spot pattern with $\mathbf{Q}_{h}=\mathbf{Q}_{s}$ are found in a well defined $(H, T)$ range for all principal axes.

sweeps were performed at several temperatures just below $T_{\mathrm{C}}=278.7(3) \mathrm{K}$ in two scattering geometries after zero $H$ cooling (ZFC): (i) Transverse geometry with the external $H$ applied in the horizontal plane along the scattering vector $\mathbf{Q}_{\mathbf{s}}$, i.e. perpendicular to the incoming neutron beam, $\mathbf{k}$, and (ii) longitudinal geometry with $H$ along the incoming beam, i.e. perpendicular to $\mathbf{Q}_{\mathbf{h}}$. In both configurations measurements were done with the sample oriented with its principal axes $(\langle 100\rangle,\langle 110\rangle$ and $\langle 111\rangle)$ along $H$. Field cooling (FC) measurements were also performed in the longitudinal geometry.

Figure 1(a) shows a SANS map in transverse geometry for $\mathbf{H} \|$ [100]. The well resolved Bragg peak corresponds to a spiral with $\mathbf{Q}_{s}=(-0.009,0,0) \AA^{-1}$ whereas the reflection at $-\mathbf{Q}_{s}$ is hardly visible. The strong peak on the left hand side flips to the right hand side for the opposite neutron polarization (not shown in Fig 1) and disappears above $T_{\mathrm{C}} \mathrm{K}$. This behavior is the consequence of neutron scattering on magnetic spiral structure, when the polarization of the neutrons is aligned along the scattering vector $\mathbf{Q}$ [33, 34. A single Bragg peak in this geometry corresponds to a mono chiral structure. Here a complete polarization analysis with analyzer after the sample is not necessary, because this peak is purely magnetic. To check the initial polarization we used a $\mathrm{MnSi}$ sample as a reference. For FeGe, the spiral vector $\mathbf{Q}_{s}=(2 \pi / a)(\xi, 0,0) \AA^{-1}$, with $a=4.70 \AA$ and $\xi=0.0069$, propagates along equivalent $\langle 100\rangle$ directions [32. Similar SANS maps have been collected with $H$ ap-

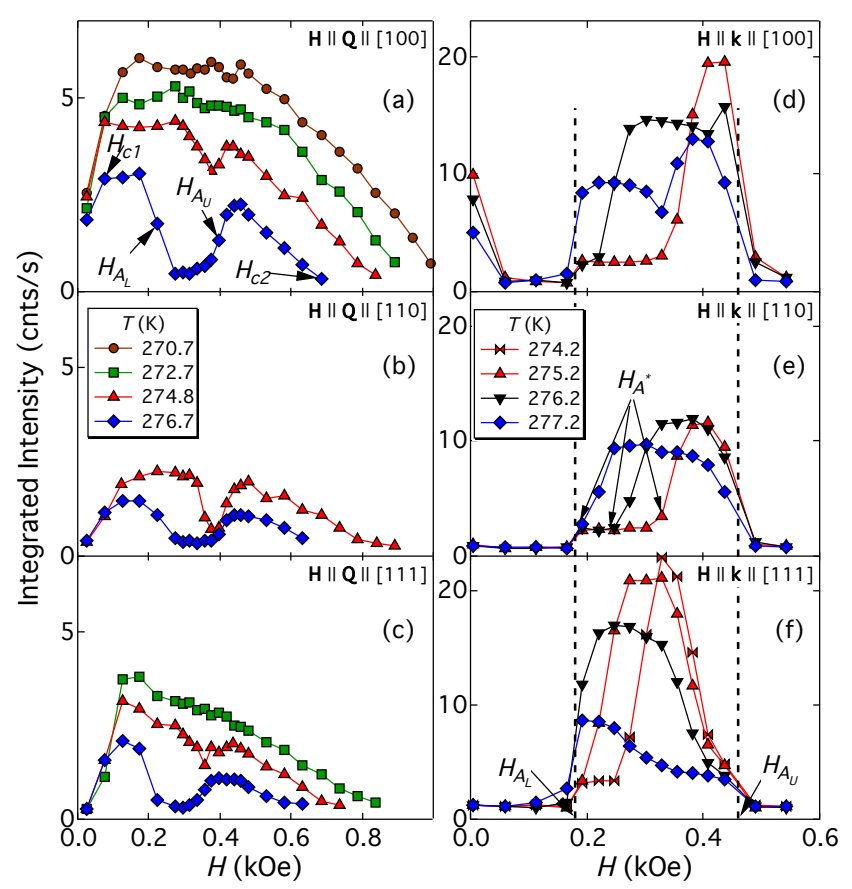

FIG. 2. (Color online) Integrated intensity vs. $H$ at various temperatures after zero $H$ cooling. (a-c) Transverse geometry. Lower $\left(H_{c 1}\right)$ and upper critical field $\left(H_{c 2}\right)$ as well as the lower and upper boundaries of the $A$ phase, $H_{A_{L}}$, and $H_{A_{U}}$, are defined for each $T$ as indicated in (a). (d-f) Longitudinal geometry. $H_{A_{L}}$ and $H_{A_{U}}$ are the same for all the temperatures and $H$ directions. The integrated intensity of the sixfold Bragg pattern shows an almost constant, plateau-like $H$ dependence, followed by a pronounced maximum above $H_{A^{*}}$, which changes with $T$ and $H$ direction. The scattering intensity of each of the six Bragg spots has a count rate very similar to that of the single Bragg spot in the transverse geometry.

plied along other principal axes (not shown) at several temperatures below $T_{\mathrm{C}}$ (for ZFC). Completely different SANS maps were observed for the longitudinal geometry $(\mathbf{H} \| \mathbf{k})$. Representative examples are shown in Fig. 1)(bd). Here a hexagonal pattern of Bragg peaks is seen at wave vector $\mathbf{Q}_{\mathbf{h}}=\mathbf{Q}_{\mathbf{s}}$. It is noteworthy that the azimuthal intensity distribution of these peaks is uniform within the statistical errors.

Figure 2 shows the integrated intensities [? ] of these detailed $H$ sweeps at selected temperatures. A strong increase in intensity at low $H$ is observed for all principal axes in the transverse geometry (Fig. 2(a-c)). A lower critical field, $H_{c 1}$, can be defined as the field where the slope of the intensity vs. $H$ dependence changes. Here spirals, with $\mathbf{Q}_{s}$ parallel to equivalent directions $\langle 100\rangle$ are aligned along a single [100] axis parallel to $H$ [21, 32]. At intermediate fields $H_{A_{L}}<H<H_{A_{U}}$ and $T>272.7 \mathrm{~K}$ (see Fig. 2(a)) a minimum in the scattering intensity occurs with almost vanishing intensity at $T=276.7 \mathrm{~K}$ (c.f. Fig. 2(a-c)). These minima are reminiscent to the reduced magnetic susceptibility observed in 


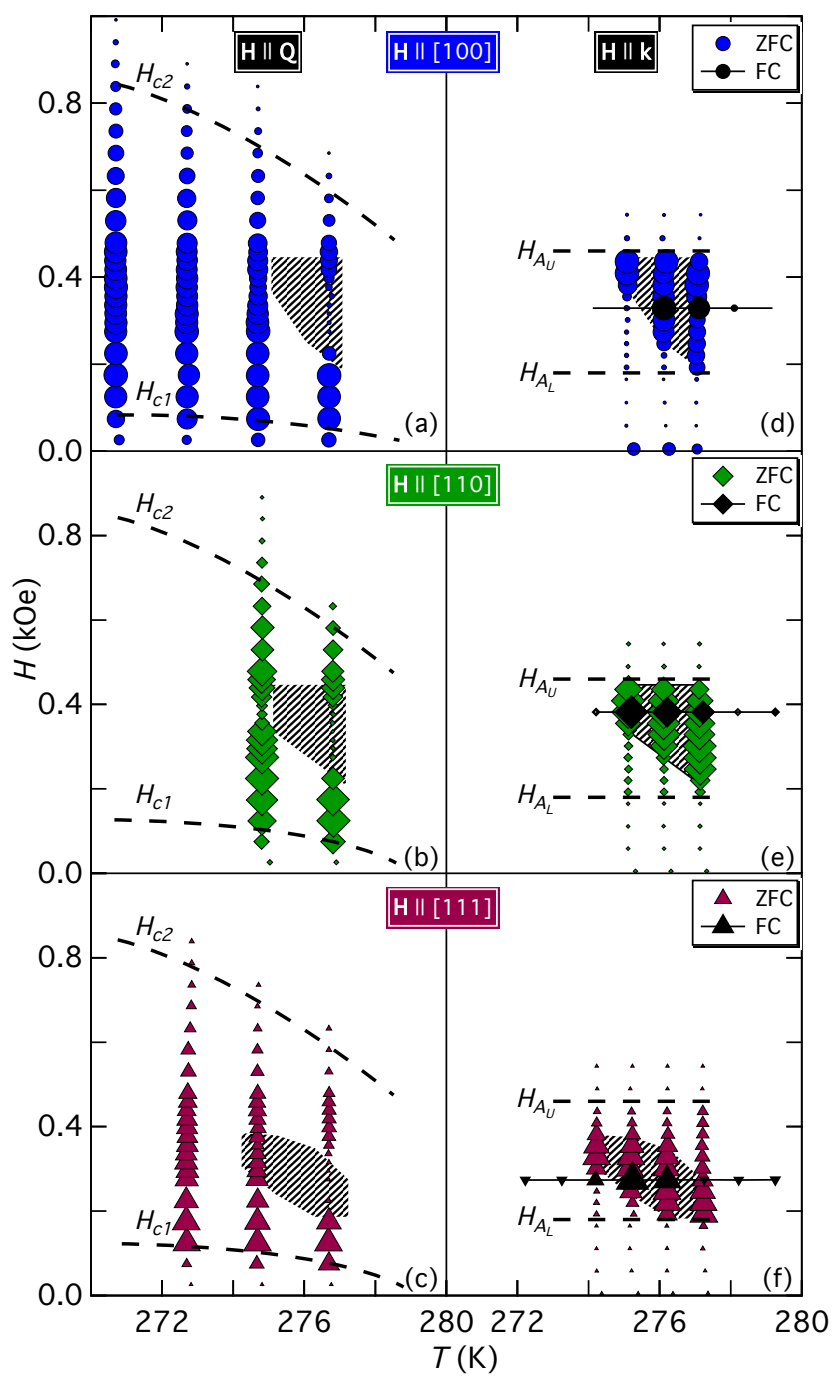

FIG. 3. (Color online) Intensity maps for transverse (left) and longitudinal (right) scattering geometry. The symbol size represents the intensity and dots indicate vanishing intensity. (a-c) Strong intensity in the major part of the phase diagram confirms the magnetic structure reported in Ref. 32. (d-f) The $H$-induced intensity variation indicates a segmentation of the $A$-phase region. The shaded regions correspond to the sixfold scattering in the longitudinal geometry. Field-cooled data (black symbols) give an idea about the $T$ width of the precursor region. The intensity at $H=0$ for $\langle 100\rangle$ is indicative for the occurrence of the helical spin structure.

$H$ sweeps across the $A$-phase region [27]. The intensity eventually disappears at $H_{c 2}$ where the field-polarized state is reached. In the longitudinal scattering geometry, however, noticeable scattering intensity is observed only for fields in the range $H_{A_{L}}<H<H_{A_{U}}$. In this region the intensity varies strongly with $H$ and $T$ (c.f. Fig. 2(df)). For $276.2 \mathrm{~K} \leq T \leq 277.2 \mathrm{~K}$ there is a $H$ range where the intensity is almost constant and followed by a region $H_{A^{*}}<H<H_{A_{U}}$, where the intensity attains a maximum. However, at just $1 \mathrm{~K}$ below this $T$ interval, a strongly reduced but still constant intensity is found in the lower $H$ region whereas the maximum at higher fields is even more pronounced (see data for $275.2 \mathrm{~K}$ in Fig. 2(d-f)). For similar temperatures the intensity in the transverse geometry has only shallow minima for the $\langle 100\rangle$ and $\langle 111\rangle$ directions whereas a well pronounced minimum is present for the $\langle 110\rangle$ direction (c.f. data at $T=274.8 \mathrm{~K}$ in Fig. 2(a-c)). Given the almost spherical shape of the crystal demagnetization and stray field effects can be ruled out. It is important to note that across the $H$ range $H_{A^{*}}<H<H_{A_{U}}$ intense single Bragg spots were observed in the transverse geometry (see below).

These observations are summarized as intensity maps in Fig. 3. In the transverse geometry (Fig. 3(a-c)) significant intensities are observed above $H_{c 1}$ where the conical phase (for $T \ll T_{\mathrm{C}}$ ) is entered [32]. Vanishing intensity above the upper critical field $H_{c 2}$ shows that the $H$-aligned state is reached. The much reduced intensity in a small $H$ interval at $T=276.7 \mathrm{~K}$ reflects the minimum found in the integrated intensity (c.f. Fig. 2(a-c)). It lays in the shaded area which indicates the phase space where the sixfold scattering was observed in the longitudinal geometry.

The occurrence of scattering intensity in both geometries unambiguously shows that the magnetic modulations have longitudinal and transversal components implying a complex magnetic structure. Furthermore, from the intensity maps of the longitudinal geometry (Fig. 3 (df)) it is obvious that the $A$-phase region is partitioned into sections with strong and weak scattering intensity. The weak-scattering section is either mainly at the low- $H$ side $(H \|\langle 100\rangle$, Fig. $3(\mathrm{~d}, \mathrm{e}))$ or at the high- $H$ side of the $A$-phase region $(H \|\langle 111\rangle$, Fig. $3(\mathrm{f}))$. Above $H_{A_{U}}$ the intensity fades away. More insight into this important aspect of a segmented $A$-phase region can be gained when the intensity maps for $H \|\langle 100\rangle$ are combined with the magnetic phase diagram established by magnetic susceptibility measurements [27].

Figure 4 shows the combined $(H, T)$ phase diagram of FeGe for $H$ along the easy axis [100]. The first observation is that the location of the $A$-phase region deduced from the SANS data are in good agreement with the phase diagram established by magnetic susceptibility measurements 27. Moreover, the segmented nature of the $A$-phase region is now confirmed by the SANS data. There is a well-localized region, the $A_{1}$ pocket, where a six-fold Bragg pattern (longitudinal geometry) and vanishing intensity (transverse geometry) were observed. Attached to it is the $A_{2}$ pocket where a hexagonal Bragg pattern (longitudinal geometry) as well as single Bragg spots (transverse geometry) were observed. The upper boundary of $A_{2}$ coincides with $H_{A_{U}}^{L g}$ and the lower boundary is close to $H_{A_{U}}^{T r}$. Thus the SANS data confirm the segmentation of the $A$-phase region in FeGe.

The $A_{2}$ pocket seems to be distinct from the $A_{1}$ as it 


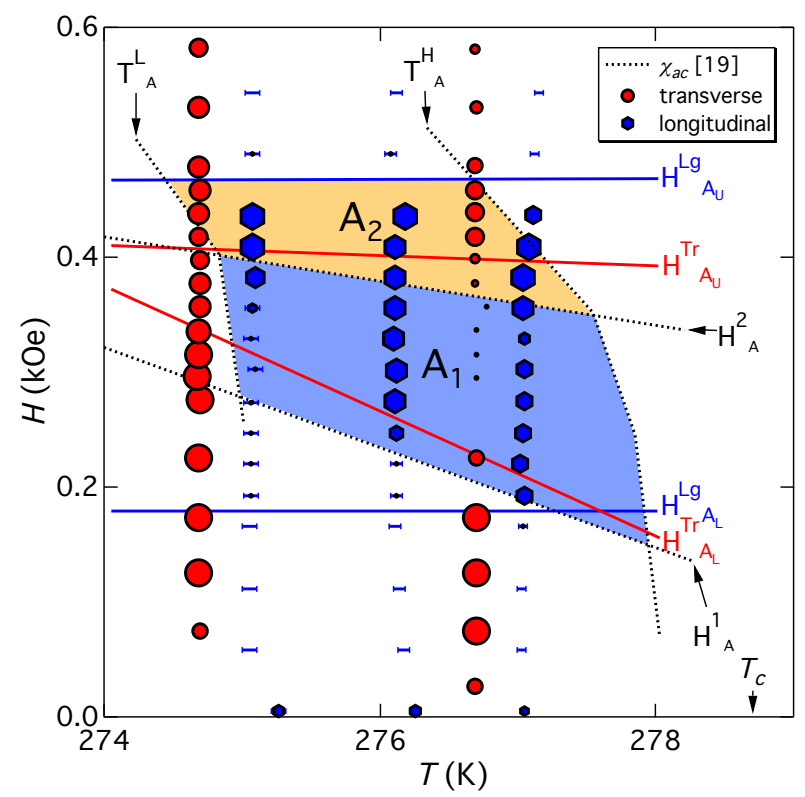

FIG. 4. (Color online) Magnetic phase diagram of FeGe based on integrated SANS intensities (colored symbols) and magnetic susceptibility data (dotted lines) 27] for $H \|$ [100]. The two pockets, $A_{1}$ and $A_{2}$, of the $A$-phase region and the phase lines are based on the magnetic-susceptibility data. Solid lines represent the lower $\left(H_{A_{L}}^{L g}\right.$ and $\left.H_{A_{L}}^{T r}\right)$ and upper $\left(H_{A_{U}}^{L g}\right.$ and $H_{A_{U}}^{T r}$ ) boundaries of the $A$ phase in the longitudinal and transverse geometry, respectively.

is characterized by the occurrence of a maximum in the intensities for the longitudinal geometry (see Fig. 2 (df)). It rules out the coexistence of a conical and a simple skyrmion-lattice phase. This points to the possibility of the existence of complex magnetic modulations outside the $A_{1}$ pocket. Therefore, it seems very likely that the SANS pattern stem from a real 3D-modulated spintexture which transforms into a cone-like structure above $H_{A_{U}}^{T r}$. This is corroborated by the $H$ dependence of the scattering intensity observed in the transverse geometry for $H_{A_{U}}^{T r}<H<H_{c 2}$. Here the intensity variation across the conical state, c.f. the curve for $T=270.7 \mathrm{~K}$ in Fig. 2(a) is very similar to the data at higher temperatures and $H_{A_{U}}^{T r}<H<H_{c 2}$. The coexistence of single and six-fold Bragg spots below $H_{A}^{(1)}$ can only be explained when either the conical helix develops some transverse modulations or a different magnetic structure nucleates within the cone phase. From the SANS data it is concluded that the magnetic structure of the $A$ phase region, except $A_{1}$, is modulated in all three spatial directions. The modulations are inhomogeneous along $H$. They rule out a magnetic texture composed of a simple triple-helix structure as suggested for $\mathrm{MnSi}$ [22]. This is supported by recent reports on $\mathrm{Cu}_{2} \mathrm{OSeO}_{3}$ where microwave-absorption [13] and SANS experiments [1] clearly indicate an inhomogeneous $A$-phase as well.
In conclusion, the detailed polarized small-angle neutron scattering data unambiguously confirm the segmentation of the $A$-phase region into several pockets. The SANS data confirm the complex phase diagram and its phase boundaries inferred from magnetic susceptibility data. Moreover, these data show that the origin and magnetic structure of the $A$ phase just below magnetic ordering is distinct from the field-driven Skyrmion lattices observed in thin films of noncentrosymmetric cubic helimagnets at temperatures deep in the magnetically ordered state.

This work was performed within the framework of a Federal Special Scientific and Technical Program (Project No.02.740.11.0874) and the state contract No 07.514.12.4003. E.M, V.D., and S.G. thank for partial support by the Russian Foundation of Basic Research (Grant No 09-02-01023, 10-02-01205-a). We acknowledge discussions with S.V. Maleyev, A.N. Bogdanov, and U.K. Rößler.

* mosqueen@pnpi.spb.ru

[1] I. E. Dzyaloshinsky, Sov. Phys. JETP 19, 960 (1964), [Zh. Eksp. Teor. Fiz. 46, 1420 (1964)].

[2] P. Bak and M. H. Jensen, J. Phys. C: Solid St. Phys. 13, L881 (1980).

[3] A. N. Bogdanov and D. A. Yablonskii, Sov. Phys. JETP 68, 101 (1989), [Zh.Eksp.Teor.Fiz. 95, 178-182 (1989)].

[4] A. Bogdanov and A. Hubert, J. Magn. Magn. Mater. 138, 255 (1994), ISSN 0304-8853.

[5] U. K. Rößler, A. N. Bogdanov, and C. Pfleiderer, Nature 442, 797 (2006).

[6] U. K. Rößler, A. A. Leonov, and A. N. Bogdanov, J. Phys.: Conf. Ser. 303, 012105 (2011).

[7] X. Z. Yu et al., Nature 465, 901 (2010).

[8] X. Z. Yu et al., Nat. Mater. 10, 106 (2011).

[9] A. Tonomura et al., Nano Lett. 12 (2012).

[10] E. A. Karhu et al., Phys. Rev. B 85, 094429 (2012).

[11] S. Seki et al., Phys. Rev. B 85, 220406 (2012).

[12] S. Seki et al., Science 336, 198 (2012).

[13] Y. Onose et al., Phys. Rev. Lett. 109, 037603 (2012).

[14] T. Adams et al., Phys. Rev. Lett. 108, 237204 (2012).

[15] S. Heinze et al., Nat. Phys. 7, 713 (2011).

[16] K. Kadowaki, K. Okuda, and M. Date, J. Phys. Soc. Jpn. 51, 2433 (1982).

[17] C. Gregory, D. Lambrick, and N. Bernhoeft, J. Magn. Magn. Mater. 104-107, 689 (1992).

[18] C. Thessieu et al., J. Phys. Condens. Matter 9, 6677 (1997).

[19] D. Lamago et al., Physica B 385-386, 385 (2006).

[20] B. Lebech et al., J. Magn. Magn. Mater. 140-144, 119 (1995).

[21] S. V. Grigoriev et al., Phys. Rev. B 73, 224440 (2006).

[22] S. Mühlbauer et al., Science 323, 915 (2009), Ibid. 333, 1381 (2011).

[23] F. Jonietz et al., Science 330, 1648 (2010), Ibid. 333, 1381 (2011).

[24] A. Neubauer et al., Phys. Rev. Lett. 102, 186602 (2009).

[25] K. Ishimoto et al., Physica B 213-214, 381 (1995). 
[26] W. Münzer et al., Phys. Rev. B 81, 041203 (2010).

[27] H. Wilhelm et al., Phys. Rev. Lett. 107, 127203 (2011).

[28] H. Wilhelm et al., J. Phys. Condens. Matter 24, 294204 (2012).

[29] A. Bauer et al., Phys. Rev. B 82, 064404 (2010).

[30] A. Bauer and C. Pfleiderer, Phys. Rev. B 85, 214418 (2012).
[31] A. A. Leonov, A. N. Bogdanov, and U. K. Rößler, arXiv:1001.1292v3 (2010).

[32] B. Lebech, J. Bernhard, and T. Freltoft, J. Phys. Condens. Matter 1, 6105 (1989).

[33] S. V. Maleyev, V. G. Bar'yahtar, and R. A. Suris, Soviet Phys. Solid State 4, 2533 (1962).

[34] M. Blume, Phys. Rev. 130, 1670 (1963). 